\title{
An Alternative Interpretation of the C-H Bond Strengths of Alkanes
}

\author{
Scott Gronert \\ Department of Chemistry and Biochemistry, \\ San Francisco State University, San Francisco, CA 94132
}

Supporting Information

\section{Index}

\begin{tabular}{lll}
\hline & Material & Page \\
\hline 1. & Gaussian reference & $\mathrm{S} 1$ \\
2. & Table S1 & $\mathrm{S} 2$ \\
3. & Table S2 & $\mathrm{S} 3$ \\
4. & Table S3 & $\mathrm{S} 4$ \\
5. & Cartesian Coordinates for species in Table S1 & $\mathrm{S} 5-\mathrm{S} 7$ \\
& $\begin{array}{l}\text { (methyl, ethyl, isopropyl, } t \text {-butyl, and their pyramidal forms) } \\
\text { 6. }\end{array}$ Cartesian Coordinates for species in Table S2 & $\mathrm{S} 8-\mathrm{S} 9$ \\
& $\begin{array}{l}\text { (silyl, trimethylsilyl, silane, and trimethylsilane) } \\
\text { 7. }\end{array}$ & Cartesian Coordinates for species in Table 2 \\
& $\begin{array}{l}\text { (isobutane, t-butyl(pyramid), t-butyl, propene, allyl, } \\
\text { allyl(pyramid), allyl, methane, methyl (pyramid), methyl) }\end{array}$ & $\mathrm{S} 10-\mathrm{S} 13$ \\
\end{tabular}

All calculations were completed with Gaussian 03. ${ }^{70}$

70. Frisch, M. J.; Trucks, G. W.; Schlegel, H. B.; Scuseria, G. E.; Robb, M. A.; Cheeseman, J. R.; Montgomery, J. A.; Vreven, T.; Kudin, K. N.; Burant, J. C.; Millam, J. M.; Iyengar, S. S.; Tomasi, J.; Barone, V.; Mennucci, B.; Cossi, M.; Scalmani, G.; Rega, N.;

Petersson, G. A.; Nakatsuji, H.; Hada, M.; Ehara, M.; Toyota, K.; Fukuda, R.; Hasegawa, J.; Ishida, M.; Nakajima, T.; Honda, Y.; Kitao, O.; Nakai, H.; Klene, M.; Li, X.; Knox, J. E.; Hratchian, H. P.; Cross, J. B.; Adamo, C.; Jaramillo, J.; Gomperts, R.; Stratmann, R. E.; Yazyev, O.; Austin, A. J.; Cammi, R.; Pomelli, C.; Ochterski, J. W.; Ayala, P. Y.; Morokuma, K.; Voth, G. A.; Salvador, P.; Dannenberg, J. J.; Zakrzewski, V. G.; Dapprich, S.; Daniels, A. D.; Strain, M. C.; Farkas, O.; Malick, D. K.; Rabuck, A. D.; Raghavachari, K.; Foresman, J. B.; Ortiz, J. V.; Cui, Q.; Baboul, A. G.; Clifford, S.; Cioslowski, J.; Stefanov, B. B.; Liu, G.; Liashenko, A.; Piskorz, P.; Komaromi, I.; Martin, R. L.; Fox, D. J.; Keith, T.; Al-Laham, M. A.; Peng, C. Y.; Nanayakkara, A.; Challacombe, M.; Gill, P. M. W.; Johnson, B.; Chen, W.; Wong, M. W.; Gonzalez, C.; Pople, J. A. Gaussian 03, Revision B04; Gaussian, Inc.: Pittsburgh, PA, 2003. 
Table S1. Pyramidalization Energies of Alkyl Radicals $(\operatorname{CCSD}(T) / 6-311+G(d, p))^{a}$

\begin{tabular}{lrrc}
\hline Radical & Fully Optimized & Pyramidal & $\Delta \mathrm{E}(\mathrm{kcal} / \mathrm{mol})$ \\
\hline Methyl & -39.7334855 & -39.7228287 & 6.7 \\
Ethyl & -78.9482314 & -78.9375255 & 6.7 \\
Isopropyl & -118.1653787 & -118.1543513 & 6.9 \\
t-Butyl & -157.3843317 & -157.373511 & 6.8 \\
\hline
\end{tabular}

${ }^{a}$ Absolute energies in hartree. No corrections for zero-point energies.

Pyramidal structures have bond angles of $109.5^{\circ}$ at the radical center.

Fully optimized (MP2/6-311+G(d,p)) aside from angle constraints. 
Table S2. Bond Energies in Silanes (G3 Level) ${ }^{a}$

\begin{tabular}{llc}
\hline Species & \multicolumn{1}{c}{ Energy } & $\Delta \mathrm{H}(\mathrm{kcal} / \mathrm{mol})$ \\
\hline$\left(\mathrm{CH}_{3}\right)_{3} \mathrm{SiH}$ & -409.562219 & \\
$\left(\mathrm{CH}_{3}\right)_{3} \mathrm{Si}$ & -408.91285 & 95.2 \\
$\mathrm{SiH}_{4}$ & -291.706952 & \\
$\mathrm{SiH}_{3}$ & -291.062211 & 92.3 \\
\hline
\end{tabular}

${ }^{a}$ Absolute energies in hartree. $\Delta \mathrm{H}$ is for $\mathrm{Si}-\mathrm{H}$ bond cleavage. Values include standard thermal corrections. 
Table S3. Energy of Methyl/Methyl Interaction in Triplet State. ${ }^{a}$

\begin{tabular}{lcc}
\hline Species & Energy & $\Delta \mathrm{E}(\mathrm{kcal} / \mathrm{mol})$ \\
\hline $\mathrm{CH}_{3}$ & -39.7201006 & \\
$\mathrm{CH}_{3} / \mathrm{CH}_{3}$ Triplet & -79.4207749 & 12.2 \\
\hline
\end{tabular}

${ }^{a} \operatorname{CCSD}(T) / 6-311+G(d, p)$ level. Absolute energies in hartree.

Geometries obtained by deleting atoms in propane $(M P 2 / 6-31+G(d))$.

No zero point energy corrections. 


\section{Cartesian Coordinates}

(Frequencies were analyzed in limited cases)

$\underline{\text { Species for Table S1 }}$

Methyl

(number of imaginary frequencies $=\mathrm{N} / \mathrm{A}$ )

C methyl

C $-0.0000125507 \quad 0.0000217385-0.0000004065$

H $-0.1823196167 \quad 0.3157868067 \quad 1.0159544892$

H $0.9007343040 .309427701-0.5079760116$

H $-0.7183393829-0.6253449388-0.5079760383$

END

Ethyl

(number of imaginary frequencies $=\mathrm{N} / \mathrm{A}$ )

C ethyl

C $0.66493753210 .0755199996-0.4322848606$

C $-0.5863850676-0.08781013150 .3657705798$

H $1.6186703795-0.2429604933-0.0309234508$

H $-0.7265447053 \quad 0.7424082778 \quad 1.0734171837$

H $-0.5670086242-1.00891718790 .9546644508$

$\mathrm{H}-1.4701684931-0.1110528185-0.2776811273$

H $0.67373665590 .6942630132-1.3203913721$

END
Isopropyl
(number of imaginary frequencies $=\mathrm{N} / \mathrm{A}$ )
C isopropyl
C $0.3116297714 \quad 0.3282324239 \quad-0.3111783213$
C $0.2439063848 \quad 0.5389363923 \quad 1.1669248406$
H $0.7339616601 \quad 1.4680944893 \quad 1.468717593$
H $0.7322280862-0.2836312468 \quad 1.7133994625$
H $-0.7961778488 \quad 0.5706235053 \quad 1.5109032825$
H $1.1718873583 \quad 0.7119973954-0.850332575$
C $-0.5024162074-0.753731624-0.9439930808$
H $-0.1156373144-1.7521764804-0.6847247371$
H $-0.5045283851-0.6770321446-2.0342593492$
H $-1.5404532487 \quad-0.7184986717-0.5942243078$
END 


$$
\begin{aligned}
& \text { t-Butyl } \\
& \text { (number of imaginary frequencies }=N / A) \\
& \text { C t-butyl } \\
& \text { C }-0.0005943968-0.17349354130 .1210494232 \\
& \text { C } 1.2812947866-0.407690863-0.6168244052 \\
& \text { H } 2.1350285396 \quad-0.45674692770 .0667028447 \\
& \text { H } 1.48346997810 .4116190734-1.3285777658 \\
& \text { H } 1.2468754625-1.3354235151-1.1969899688 \\
& \text { C }-0.0006021437 \quad 0.8641303663 \quad 1.2006547465 \\
& \text { C }-1.2804708055-0.4015102778-0.6222399549 \\
& \text { H }-1.47662906720 .419796077-1.3333718479 \\
& \text { H -2.1370879137 }-0.44823901470 .0578612835 \\
& \text { H }-1.2474859356-1.328449845-1.2037321822 \\
& \text { H } 0.88664053640 .7822220297 \quad 1.8366479902 \\
& \text { H } \quad-0.8902488159 \quad 0.7851595678 \quad 1.8337003816 \\
& \text { H } 0.0016725721 \quad 1.8814484493 \quad 0.7719204063 \\
& \text { END }
\end{aligned}
$$$$
\text { Methyl (pyramidal) }
$$$$
\text { (number of imaginary frequencies }=N / A)
$$$$
\text { C methyl }
$$$$
\text { C }-0.05697222750 .0986787927-0.0403031398
$$$$
\text { H }-0.05732305420 .0992865963 \quad 1.0490025419
$$$$
\text { H } 0.96997358410 .0991082748-0.4035919142
$$$$
\text { H }-0.5708171646-0.7904676274-0.4035917886
$$$$
\text { END }
$$

Ethyl (pyramidal)

(number of imaginary frequencies $=N / A$ )

C ethyl

C $0.6521857106-0.0447303528-0.5112717048$

C $-0.573736614-0.0645525314 \quad 0.3738621611$

H $1.5500800737 \quad-0.05012965160 .1096359805$

$\begin{array}{lllll}\mathrm{H} & -0.5578586434 & 0.8289217465 & 1.0124474393\end{array}$

H $-0.6003512286-0.9377542372 \quad 1.0312435377$

H $-1.5039909184-0.0409533827-0.2002794058$

H $0.6414261373 \quad 0.8556128301-1.1285902888$

END 

Isopropyl (pyramidal)
$($ number of imaginary frequencies $=N / A)$
C isopropyl
$\begin{array}{llll}\text { C } & 0.2474851284 & 0.4812521147 & -0.3822048215\end{array}$
$\begin{array}{llll}\text { C } & 0.2324698792 & 0.4905234052 & 1.1315317662\end{array}$
H 0.74712598591 .35618360131 .5589535084
H $0.7457984719-0.4120338785 \quad 1.4922730316$
H $\quad-0.7886319757 \quad 0.4695897163 \quad 1.5254169412$
H $1.28235017180 .4632932765-0.7370876199$
C $-0.4812866185-0.7457383678-0.8872750822$
H $0.0369779568-1.6397462839-0.5125727593$
H $-0.5037900104-0.8104651543-1.9791687901$
H $-1.511840935-0.7830441904-0.5201254874$
END
t-Butyl (pyramidal)
(number of imaginary frequencies $=\mathrm{N} / \mathrm{A}$ )
C t-butyl
C $-0.0013775039-0.37285645340 .2597393449$
C $1.2381078106-0.3700732052-0.6121258264$
H $2.1612076767-0.3518451763-0.0237061095$
H $1.22071168050 .5376103315-1.2337977493$
H $1.2757841087-1.2325905128-1.2854866507$
C $-0.00067100520 .8579711047 \quad 1.1440150534$
C $-1.2369842735-0.3647383642-0.6175704596$
H $-1.21526358270 .5451368518-1.2358767622$
H -2.1627307986 $-0.3468603951-0.033265734$
H $-1.2731576623-1.2248470434-1.2940553945$
H 0.88483863210 .90908101641 .7858169331
H $\quad-0.89051679950 .9148178035 \quad 1.7792975769$
H $0.00467657751 .7476786337 \quad 0.4967252165$
END 


\section{Species for Table S2}

Trimethylsilane

(number of imaginary frequencies $=0$ )

C trimethylsilane

H $\quad 0.0 .1 .8759673838$

Si $\quad 0.0 .0 .3818426149$

C $\quad 1.7824216678-0.0000000062 \quad-0.2242054685$

C $\quad-0.8912108286 \quad 1.5436224477 \quad-0.2242054685$

C $\quad-0.8912108393-1.5436224415-0.2242054685$

$\mathrm{H} \quad-0.3967630456-2.45340148990 .1278393073$

$\mathrm{H} \quad-0.9115124757-1.5787859069-1.3177004678$

$\mathrm{H} \quad-1.926326504 \quad-1.57030760840 .1278393073$

$\begin{array}{lllll}\mathrm{H} & 2.3230895327 & -0.8830938843 & 0.1278393073\end{array}$

$\begin{array}{lllll}\mathrm{H} & 2.3230895388 & 0.8830938682 & 0.1278393073\end{array}$

$\mathrm{H} \quad 1.8230249404-0.0000000063-1.3177004678$

H $\quad-0.3967630286 \quad 2.4534014927 \quad 0.1278393073$

H $\quad-1.9263264931 \quad 1.57030762180 .1278393073$

H $\quad-0.9115124647 \quad 1.5787859132 \quad-1.3177004678$

END

Trimethylsilyl

(number of imaginary frequencies $=0$ )

C trimethylsilyl

C $\quad 1.34865188420 .2890305388-1.1548529487$

Si $\quad 0.2440937715-0.15978996440 .3105365896$

C $\quad-1.0194569865-1.4688765121-0.1979777845$

C $\quad-0.6341746028 \quad 1.37949340940 .9648349414$

$\begin{array}{lllll}\mathrm{H} & 0.0822073528 & 2.1433438981 & 1.2778644281\end{array}$

$\mathrm{H} \quad-1.26751178891 .14141382 \quad 1.8232405882$

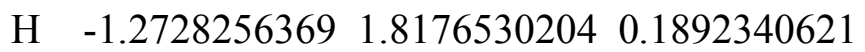

H $\quad 2.0963890028 \quad 1.0356374258 \quad-0.8753435678$

$\mathrm{H} \quad 0.7512913026 \quad 0.7044815632-1.9745946725$

$\mathrm{H} \quad 1.8767950568-0.5878090995-1.5380959179$

H $\quad-0.5287617936-2.3735148118-0.5660890586$

H $\quad-1.6661315081-1.0900326439-0.9977934499$

H $\quad-1.6588865578-1.75199828710 .6420400841$

END 
Silane

(number of imaginary frequencies $=0$ )

C silane

$\mathrm{H} \quad-0.8559479254 \quad 0.85594792540 .8559479254$

Si 0.0 .0$.

H $\quad 0.8559479254 \quad-0.85594792540 .8559479254$

H $\quad 0.8559479254 \quad 0.8559479254 \quad-0.8559479254$

H $\quad-0.8559479254 \quad-0.8559479254 \quad-0.8559479254$

END

Silyl

(number of imaginary frequencies $=0$ )

C silyl

$\begin{array}{lllll}\mathrm{Si} & -0.0458722937 & 0.0458722937 & 0.0458722937\end{array}$

H $\quad 0.7908013821 \quad-0.7908013821 \quad 0.9393906517$

H $\quad 0.7908013821 \quad 0.9393906517 \quad-0.7908013821$

H $\quad-0.9393906517 \quad-0.7908013821 \quad-0.7908013821$

END

$\underline{\text { Species for Table S3 }}$

Methyl/Methyl Triplet

(number of imaginary frequencies $=\mathrm{N} / \mathrm{A}$ )

$\mathrm{C} \mathrm{CH} 3 / \mathrm{CH} 3$ triplet

C $0.27163679490 .4477627473 \quad 1.1840579778$

H 0.79407585531 .33437217661 .5576187055

H $0.7822621994 \quad-0.436287263 \quad 1.5813738061$

H $-0.7446653211 \quad 0.4583664329 \quad 1.59304734$

C $-0.4844202098-0.8152383693-0.881377895$

H $0.0052966881-1.7347471872-0.5422514945$

H $-0.500048796-0.8263757951-1.9760398273$

H $-1.5216186839-0.8401810866-0.5295925126$

END

Methyl

(number of imaginary frequencies $=\mathrm{N} / \mathrm{A}$ )

$\mathrm{C} \mathrm{CH} 3$

$\begin{array}{llll}\text { C } & 0.2716367949 & 0.4477627473 & 1.1840579778\end{array}$

H 0.79407585531 .33437217661 .5576187055

H $0.7822621994 \quad-0.436287263 \quad 1.5813738061$

H $-0.7446653211 \quad 0.4583664329 \quad 1.59304734$

END 
Species from Table 2

Isobutane

(number of imaginary frequencies $=\mathrm{N} / \mathrm{A}$ )

$\operatorname{CCSD}(\mathrm{T})=-158.0450427$

C isobutane

$\begin{array}{llll}\text { C } & 1.44043551 & 0 . & -0.062717222\end{array}$

C 0. 0. 0.445742291

C $-0.720217755-1.247453745-0.062717222$

C $-0.720217755 \quad 1.247453745-0.062717222$

H 0.0 .1 .538696605

H $-0.227799373-2.1589928720 .277492506$

H $-0.724857377-1.255489805-1.153747421$

H $-1.755842987-1.276776480 .277492506$

H $-0.724857377 \quad 1.255489805-1.153747421$

$\begin{array}{lllll}\mathrm{H} & -0.227799373 & 2.158992872 & 0.277492506\end{array}$

H $-1.755842987 \quad 1.27677648 \quad 0.277492506$

H 1.449714753 0. -1.153747421

H $1.98364236 \quad-0.8822163920 .277492506$

H 1.983642360 .8822163920 .277492506

END

t-Butyl

(number of imaginary frequencies $=\mathrm{N} / \mathrm{A}$ )

$\operatorname{CCSD}(\mathrm{T})=-157.3838903$

C t-butyl radical

C $1.479055747 \quad 0.0 .028531848$

C 0. 0. 0.241371018

$\begin{array}{llll}\text { C } & -0.739527874 & -1.280899811 & 0.028531848\end{array}$

$\begin{array}{lllll}\text { C } & -0.739527874 & 1.280899811 & 0.028531848\end{array}$

H $-0.206705162-2.1258669420 .465636495$

H $-0.865646946-1.499344499-1.040261809$

H $-1.737702172-1.2419453980 .465636495$

H $-0.8656469461 .499344499-1.040261809$

$\begin{array}{llll}\mathrm{H} & -0.206705162 & 2.125866942 & 0.465636495\end{array}$

H $-1.737702172 \quad 1.241945398 \quad 0.465636495$

H $1.731293891 \quad 0 .-1.040261809$

H $1.944407334 \quad-0.8839215440 .465636495$

H $1.9444073340 .883921544 \quad 0.465636495$

END 

t-Butyl (pyramidal)
(number of imaginary frequencies $=\mathrm{N} / \mathrm{A}$ )
$\operatorname{CCSD}(\mathrm{T})=--157.3730678$
C t-butyl radical pyramid
C 1.4262961130 .0 .065978
C 0.0 .0 .569523095
C $-0.713148083-1.235208657 \quad 0.065978$
C $-0.713148083 \quad 1.235208657 \quad 0.065978$
$\begin{array}{llll}\mathrm{H} & -0.225497084 & -2.156296631 & 0.386443887\end{array}$
H $-0.704347393-1.219965502-1.028706806$
H $-1.754659067-1.273434552 \quad 0.386443887$
H $-0.704347393 \quad 1.219965502-1.028706806$
$\begin{array}{lllll}\mathrm{H} & -0.225497084 & 2.156296631 & 0.386443887\end{array}$
H $-1.754659067 \quad 1.273434552 \quad 0.386443887$
H 1.4086948390 . -1.028706806
H $1.980156203 \quad-0.8828620790 .386443887$
H $1.980156203 \quad 0.8828620790 .386443887$
END

Propene

(number of imaginary frequencies $=\mathrm{N} / \mathrm{A}$ )

$\operatorname{CCSD}(\mathrm{T})=-117.6023974$

C propene

H -1.662515010 .1210193930 .947168779$

C $-1.145807008-0.094300241 \quad 0.012321516$

H $-1.079755294-1.171916495-0.12648468$

$\begin{array}{llll}\text { C } & 0.236927201 & 0.491209979 & 0.057234726\end{array}$

H $-1.699218515 \quad 0.343064814-0.818268141$

$\begin{array}{llll}\text { C } & 1.365058745 & -0.217647801 & -0.055812139\end{array}$

H $0.304613596 \quad 1.5659964590 .191566784$

H $1.344268545-1.291248078-0.190977016$

H $2.336427794 \quad 0.253822023-0.016749828$

END 

Allyl
(number of imaginary frequencies $=\mathrm{N} / \mathrm{A}$ )
$\operatorname{CCSD}(\mathrm{T})=-116.9553382$
C allyl radical
C $1.224085357 \quad 0.104386355-0.003559985$
H 1.3315208291 .1791524090 .002207303
C $-0.022933208-0.479588405-0.002781354$
H $2.126107506-0.485829307-0.010123844$
$\begin{array}{llll}\text { C } & -1.208473895 & 0.221551434 & 0.005409724\end{array}$
H $-0.074971099-1.562178183-0.008601455$
H $-1.212221844 \quad 1.3017123950 .009941066$
H $-2.163113648-0.2792066970 .007508492$
END

\begin{abstract}
Allyl (pyramidal)
(number of imaginary frequencies $=\mathrm{N} / \mathrm{A}$ )

$\operatorname{CCSD}(\mathrm{T})=-116.9354719$

$\mathrm{C}$ allyl radical pyramid

$\begin{array}{llll}\text { C } & 1.266426449 & 0.120495614 & 0.286808219\end{array}$

H $1.2608711491 .133899467-0.107592691$

C $-0.017765583-0.4768698110 .086526102$

H $2.023256294-0.465117637-0.228314369$

C $-1.1690390420 .218048018 \quad 0.003331116$

H $-0.07025502-1.5590367230 .038448678$

H $-1.173956102 \quad 1.2969257770 .074805096$

H $-2.119538144-0.268344704-0.154012203$

END
\end{abstract}

Methane

(number of imaginary frequencies $=\mathrm{N} / \mathrm{A}$ )

$\operatorname{CCSD}(\mathrm{T})=-40.4059721$

C methane

H -0.6267148520 .6267148520 .626714852$

C 0. 0. 0 .

H $0.626714852 \quad-0.6267148520 .626714852$

H $0.6267148520 .626714852-0.626714852$

H $-0.626714852-0.626714852-0.626714852$

END 
Methyl

$\operatorname{CCSD}(T)=-39.733342$

(number of imaginary frequencies $=N / A)$

C methyl radical

H 1.0745321050 . -0.000181508

C 0.0 .0 .00054447

H $-0.537266052-0.930572095-0.000181508$

H $-0.5372660520 .930572095-0.000181508$

END

Methyl (pyramidal)

(number of imaginary frequencies $=\mathrm{N} / \mathrm{A}$ )

$\operatorname{CCSD}(\mathrm{T})=-39.7226785$

C methyl radical pyramid

H 1.022426427 0. -0.090253042

C 0.0 .0 .270759074

H $-0.51121324-0.885447267-0.090253042$

$\begin{array}{llll}\mathrm{H} & -0.51121324 & 0.885447267 & -0.090253042\end{array}$

END 